\title{
Identifying Orthogonal Solvents for Solution Processed Organic Transistors
}

\author{
Abhinav M. Gaikwad, ${ }^{\# *}$ Yasser Khan, ${ }^{\#}$ Aminy E. Ostfeld, ${ }^{\#}$ Shishir Pandya, ${ }^{+}$Sameer Abraham ${ }^{\#}$ and Ana \\ Claudia Arias ${ }^{\# *}$
}

\begin{abstract}
Identification of solvents for dissolving polymer dielectrics and organic semiconductors is necessary for the fabrication of solution-processed organic field effect transistors (OFETs). In addition to solubility and printability of a solvent, orthogonality is particularly important when forming multilayer structure from solutions. Currently, the process of finding orthogonal solvents is empirical, and based on trial-and-error experimental methods. In this paper, we present a methodology for identifying orthogonal solvents for solution-processed organic devices. We study the accuracy of Hildebrand and Hansen solubility theories for building solubility boundaries for organic semiconductor (Poly(2,5-bis(3-hexadecylthiophen-2yl)thieno[3,2-b]thiophene (PBTTT) and polymer dielectrics (Poly(methyl methacrylate) (PMMA), Polystyrene (PS)). The Hansen solubility sphere for the organic semiconductor and polymer gate dielectrics are analyzed to identify solvents that dissolve PMMA and PS, but are orthogonal to PBTTT. Top gate/bottom contact PBTTT based OFETs are fabricated with PMMA gate dielectric processed with solvents that are orthogonal and non-orthogonal to PBTTT. The non-orthogonal solvents swell the semiconductor layer and increase their surface roughness.
\end{abstract}

\section{INTRODUCTION}

Solution-processed organic semiconductors have enabled the fabrication of large-area electronics by depositing electronic materials with printing methods such as gravure, ${ }^{1}$ inkjet, ${ }^{2}$ flexographic ${ }^{3}$ and offset. $^{4,5}$ Over the past two decades, efforts in molecular engineering, ${ }^{6-8}$ understanding of interfacial effects, ${ }^{9}$ and control over processing conditions, ${ }^{10,11}$ have led to significant improvements in the electrical characteristics of organic field effect transistors (OFETs). ${ }^{12,13}$ OFETs are ideal for applications such as smart tags, sensors, and active-matrix backplanes for displays and imagers, where the electronic components are less dense and distributed over large area. ${ }^{12,14-17}$

The typical structure of OFET consists of a source and drain electrode in contact with conjugated organic semiconductor layer, which is separated from the gate electrode by an insulating dielectric layer. The gate voltage modulates the charge density in the semiconductor layer and the voltage bias between the source and drain electrode controls the flow of current. ${ }^{18}$ Early demonstrations of OFETs were hybrid devices where $\mathrm{Si}$ wafers with thermally grown $\mathrm{SiO}_{2}$ gate insulator were used as common gate to study the electronic properties of vapor deposited small molecules, and polymeric semiconductors. ${ }^{7,19,20}$ In order to take full advantage of low cost fabrication processes and mechanical flexibility of organic semiconductor, solution processable polymer dielectrics are necessary. Insulating polymers such as Poly(methyl methacrylate) (PMMA), Polystyrene (PS), Polyvinylpyrrolidone (PVP) and CYTOP have been used extensively as gate dielectric in OFETs. ${ }^{21,22}$ The choice of gate dielectric is important because performance and stability of OFETs is sensitive to the nature of the semiconductor-dielectric interface. ${ }^{9,23}$ Furthermore, 
the mobility of OFETs depends on the molecular ordering of the semiconductor layer, ${ }^{7,24}$ presence of chemical and physical traps, ${ }^{23,25}$ and alignment of the energy levels between the contacts and semiconductor. $^{26,27}$

OFETs are fabricated with four different architectures, bottom gate/bottom contact, bottom gate/top contact, top gate/ bottom contact and top gate/top contact. ${ }^{18}$ Bottom gate architecture has been widely used by the research community as the semiconductor can be deposited as the last wet processing step during the fabrication process, preventing the swelling of the semiconductor layer by the polymer dielectric solvent. In addition, the surface energy of the dielectric surface can be modified to improve the morphology of the semiconductor layer. ${ }^{24}$ Devices with top gate architectures are advantageous as compared to bottom gate architectures due to easy of fabrication, low contact resistance and environmental stability. ${ }^{12,18}$ The source and drain electrodes in top gate OFETs can be patterned on substrates using high-resolution techniques such as photolithography, or printed using high-speed, roll-to-roll printing methods such as gravure, flexographic or offset, followed by the deposition of the semiconductor, dielectric layer and top electrode. Due to the staggered configuration, OFETs with top gate / bottom contact architecture have low contact resistance in comparison to the other architectures. ${ }^{28-30}$ In addition, the dielectric and top electrode provide an autoencapsulation for the semiconductor layer, which can improve its environmental stability. ${ }^{21}$

Even with these advantages, OFETs with top gate / bottom contact architecture have been difficult to implement. Finding a solvent that dissolves the polymer dielectric, but is orthogonal (solvent that dissolves one material and does not dissolve the other) to the organic semiconductor is a challenge and for most organic semiconductors only a handful of suitable orthogonal solvents have been successfully identified. ${ }^{31-35}$ Solubility models such as Hansen and Hildebrand theory have been used extensively by the organic solar cell community to find green, non-chlorinated solvents for processing the active layer. ${ }^{36-39}$ Similar methods have not been fully utilized for the fabrication of OFETs. ${ }^{31,40}$ In this work, we present a methodology to identify orthogonal solvents for solution processed devices. We study the accuracy of Hildebrand and Hansen solubility theories for defining solubility boundaries for organic semiconductor (Poly(2,5-bis(3hexadecylthiophen-2-yl)thieno[3,2-b]thiophene (PBTTT) and polymer dielectrics (PMMA and PS). With the goal of fabricating top gate/bottom contact PBTTT based OFETs, the solubility data was used to identify solvents that dissolve the polymer gate dielectrics but are orthogonal to the semiconductor (Figure 1). Top gate/bottom contact PBTTT based OFETs were fabricated with polymer gate dielectric dissolved in solvents that are orthogonal and non-orthogonal towards PBTTT. The effect of orthogonality of the polymer dielectric solution on the morphology of the PBTTT layer was studied.

\section{EXPERIMENTAL SECTION}

Poly(2,5-bis(3-hexadecylthiophen-2-yl)thieno[3,2-b]thiophene $\quad$ (PBTTT, $\quad$ Merck), 6,13Bis(triisopropylsilylethynyl)Pentacene (TIPS-pentacene, Sigma Aldrich), PMMA (Sigma Aldrich, Mol. Wt. = 120,000) and PS (Sigma Aldrich, Mol. Wt. = 280,000) were used as received without further purification. $2 \mathrm{mg} / \mathrm{mL}$ solutions of the organic semiconductors (PBTTT and TIPS-pentacene) and $20 \mathrm{mg} / \mathrm{mL}$ solutions of the polymer dielectric (PMMA and PS) in thirty-three selected solvents were prepared. The solvents used in this study are list in Table 1. In order to facilitate the dissolution of the polymers, the solutions were heated overnight at $70^{\circ} \mathrm{C}$.

Accurate estimation of the solubility boundaries for the organic semiconductors and polymer dielectrics is essential to identify solvents that solubilize the polymer dielectrics but are orthogonal (nonsolvent) to the organic semiconductors. The solubility boundary for the organic semiconductor was defined 
to represent the boundary between orthogonal and non-orthogonal solvents. The orthogonal solvents were defined as 'bad solvents' and the remaining solvents were defined as 'good solvents'. The solubility boundary for the polymer dielectric was defined to identify solvents that dissolve the polymer dielectric. Solvents that were able to dissolve $20 \mathrm{mg} / \mathrm{mL}$ of the polymer dielectrics were classified as 'good solvents' and the remaining solvents were classified as 'bad solvents'.

OFETs were fabricated using in top gate / bottom contact configuration on a glass substrate. The substrate (Corning 1737) was cleaned in a soap solution (Decon 90) for one hour followed by ultrasonication with acetone and isopropanol for 10 minutes. The substrate was treated in oxygen plasma for 5 minutes before evaporating gold $(30 \mathrm{~nm})$ with $\mathrm{Cr}(5 \mathrm{~nm})$ adhesion layer. To deposit this layer, the $\mathrm{Cr} / \mathrm{Au}$ layer was patterned using standard lithography technique to form the source and drain contacts. A self-assembled monolayer of PFBT was formed on the gold electrode to increase the work function of the electrode and decrease the contact resistance. The substrate with the $\mathrm{Cr} / \mathrm{Au}$ electrodes was immersed in a solution of 10 $\mathrm{mM}$ solution of pentafluorobenzenethiol (PFBT) in ethanol for 2 minutes. A solution of PBTTT was prepared by mixing $5 \mathrm{mg} / \mathrm{mL}$ of PBTTT in dichlorobenzene. Due to poor solubility of PBTTT, the solution turned into a gel when cooled to room temperature. In order to solubilize PBTTT, the solution was heated to $85^{\circ} \mathrm{C}$ for 20 minutes before spin coating. A hot solution of PBTTT was spin coated on the glass substrate at 2000 RPM for 1 minute in a glove box with low oxygen and moisture levels $(<2 \mathrm{ppm})$. The substrate was heated on a hot plate at $110^{\circ} \mathrm{C}$ for $1 \mathrm{hr}$ to remove residual solvents followed by thermal annealing at high temperature to improve molecular ordering. The substrate was placed on a hot plate at $175^{\circ} \mathrm{C}$ for $10 \mathrm{~min}$ and the hot plate was cooled at $10^{\circ} \mathrm{C} / \mathrm{min}$ to room temperature. A polymer dielectric layer was formed by spin coating at solution of PMMA $(60 \mathrm{mg} / \mathrm{mL})$ in various solvents at $1500 \mathrm{RPM}$ for 1 minute in a glove box followed by heating the substrate on a hot plate at $115^{\circ} \mathrm{C}$ for $2 \mathrm{hr}$ to remove residual solvent. The PMMA solutions were prepared by dissolving PMMA in various solvents by heating the solution overnight on a hot plate at $70^{\circ} \mathrm{C}$. The dielectric solutions were filtered twice with a $0.1 \mu \mathrm{m}$ filtrate before spin coating. The gate contact was formed by evaporating $30 \mathrm{~nm}$ gold on the dielectric layer with a shadow mask.

The electrical characterizations of the fabricated OFETs were measuring using semiconductor characterization system (Agilent Technologies, B1500A) in atmosphere under ambient condition. All measurements were performed within two hours of exposing the device to atmospheric conditions. A total of 8 to 10 devices were measured for each combination of polymer dielectric and organic semiconductor. Highresolution topographic studies of the films were carried out using atomic force microscope (MFP-3D, Asylum Research) in the normal tapping mode under ambient conditions. The Hansen solubility sphere was fitted to the solubility data using a custom made software. Detailed description about the software and operating instructions are giving in the supplementary section. In short, based on the results of the solubility study, the solvents were categorized as 'good' or 'bad' solvents. A convex hull was created by randomly picking three good solvents from the outer regions of the Hansen space. A sphere passing through three good solvents was fitted, and the radius and center of the sphere was extracted. If all other good solvents were present inside the sphere, the center of the sphere represented the Hansen parameters for the material and the radius of sphere represented the radius of interaction for the material. If any good solvents were present outside the sphere, a new set of three good solvents were picked and the process was repeated until all good solvents were enclosed inside the sphere.

\section{RESULTS AND DISCUSSION}

\subsection{Hildebrand and Hansen Solubility Theories}


In Hildebrand theory, each material is given a solubility number $(\delta)$, which is commonly referred as 'Hildebrand Solubility Parameter'. ${ }^{41-43}$ The solubility parameter gives a numerical value for the total cohesive attractive interactions within a material due to Van der Waals forces. For a liquid, the solubility parameter is defined as the square root of the total cohesive energy density (energy required in cal./cc to convert a substance from liquid to gaseous state).

$$
\delta=\left(\frac{E}{V}\right)^{0.5}
$$

$\mathrm{V}$ is the molar volume of pure solvent and $\mathrm{E}$ is the energy of vaporization. The solubility parameter has units of $\mathrm{MPa}^{1 / 2}$.

Heat of vaporization and solubility are related to each other as the energy required to break all the attractive bonds within a substance while converting it from liquid to vapor state is similar to the change in free energy required while dispersing two materials together. Materials with similar values of solubility parameter are likely to be miscible with each other and a large difference in solubility parameter would indicate poor miscibility. Hildebrand parameters for most commonly used organic solvents have been experimentally measured and are readily available in the literature. ${ }^{43}$ Solubility parameter for polymers cannot be directly measured as they decompose before reaching their boiling point. Instead, the solubility parameters for polymers are extracted by measuring the degree of swelling or dissolution in series of solvents with increasing Hildebrand value. The polymers are assigned a solubility parameter value based on solvent that swells or dissolves them to the highest extent. The region encompassing all the good solvents for a material represents the solubility window for the material and the bad solvents are present outside the solubility window.

Even with the apparent simplicity of Hildebrand solubility theory, the solubility prediction can be incorrect under certain conditions. Van der Waals attractive forces are an additive combination of three different forces: dispersion forces, dipole forces and hydrogen bonding forces. The Hildebrand solubility theory combines all the constituent forces into a single number. Solvents with similar Hildebrand parameter number can have large differences in their individual cohesive interactions, which can lead to a false prediction of miscibility and orthogonality when the prediction is based solely on the summation of the all the attractive forces.

Hansen solubility theory provides a more accurate estimation of solubility by dividing the Hildebrand solubility parameter into its constitutive interaction parameters. The total cohesive energy is given as the addition of the cohesive energy due to dispersion force, polar forces and hydrogen bonding force. $^{44,45}$

$$
\mathrm{E}=\mathrm{E}_{\mathrm{D}}+\mathrm{E}_{\mathrm{P}}+\mathrm{E}_{\mathrm{H}}
$$

Dividing equation (2) by the molar volume and using Hildebrand formalization, we get the following equations.

$$
\begin{gathered}
\mathrm{E} / \mathrm{V}=\mathrm{E}_{\mathrm{D}} / \mathrm{V}+\mathrm{E}_{\mathrm{P}} / \mathrm{V}+\mathrm{E}_{\mathrm{H}} / V \\
\delta_{\mathrm{T}}^{2}=\delta_{\mathrm{D}}^{2}+\delta_{\mathrm{P}}^{2}+\delta_{\mathrm{H}}^{2}
\end{gathered}
$$

The square of the Hildebrand parameter is equal to the addition of squares of the solubility parameters for the dispersion, polar and hydrogen-bonding component. The Hansen parameters for a material are represented as a point in a three-dimensional space (Hansen Space) with the dispersion, polar and hydrogen bonding components plotted along the three axes. The Hansen parameters for most organic solvents are available in the literature. The Hansen parameters for polymers are extracted by dissolving the polymer in a set of $30-40$ solvents that are well dispersed in the Hansen Space. ${ }^{43}$ Solvents are classified as good or bad solvent based on the degree of swelling or dissolution of the polymer. The good solvents aggregate together and form a shape of an ellipsoid in the Hansen space. Based on experimental analysis, 
Hansen suggested multiplying the dispersion axis by a factor of two to transform the space representing good solvents from an ellipsoid to a sphere, making it easy to visualize the data. ${ }^{43}$ Computer software can be use to fit a sphere to the region representing good solvents (software description given in the supplementary section). The center of the sphere represents the Hansen solubility parameters $\left(\delta_{D}, \delta_{P}, \delta_{H}\right)$ for the polymer and the radius is called as the 'Radius of Interaction'. The region within the sphere encompasses the good solvents and the region outside the sphere represents the bad solvents. Once the Hansen parameters for a polymer are extracted, the solubility of any solvent can be predicted by measuring its distance from the center of the sphere. If the distance is less than the radius of interaction, the solvent is a good solvent and if the distance is greater than the radius, the solvent is a bad solvent. The distance of a solvent relative to the radius of the sphere is commonly referred to as RED number (Relative Energy Difference).

$$
\mathrm{RED}=\frac{\mathrm{R}_{\mathrm{a}}}{\mathrm{R}}=\frac{\text { Distance from center }}{\text { Radius of sphere }}
$$

RED value less than one indicates a good solvent and above one indicates a bad solvent.

\subsection{Solubility Theories Applied to Organic Semiconductors}

Solutions of PBTTT with concentration of $2 \mathrm{mg} / \mathrm{mL}$ in thirty-three selected solvents were prepared (Table 1). The solutions were stirred overnight at $70^{\circ} \mathrm{C}$ and classified by visual inspection. Solvents that were completely orthogonal to the semiconductor were classified as 'bad solvents' and the remaining solvents (non-orthogonal) were classified as 'good solvents'. Figure $\mathbf{2}$ shows the optical image of supernatant of the solvents that showed a range of solubility towards PBTTT (Grade A-E). The orthogonal solvents were rated as Grade-E. The good solvents were further classified based on the extent of dissolution of the semiconductor. Solvents that dissolved the semiconductor completely were rated as Grade-A. These solvents can be used for depositing the semiconductor layer. Solvents with small quantities of undissolved semiconductor were rated as Grade-B. Solvents with large quantities of undissolved semiconductor were rated as Grade-C. Solvents that had very poor solubility and swelled the semiconductor by a small extent (observed by visual inspection) were rated as Grade-D. Results of the solubility test are listed in Table 1.

Results from the solubility test for PBTTT was analyzed using Hildebrand and Hansen solubility theories. Figures 3A show the solubility data for PBTTT plotted using Hildebrand methodology. The solvents are arranged according to their Hildebrand parameter and labeled as 'good solvent' or 'bad solvent'. Grade A to D solvents were considered as good solvents and grade E solvents were considered as bad solvents. PBTTT interacted with solvents with solubility parameter ranging from $17.8-20.5 \mathrm{MPa}^{0.5}$. Out of the thirty-three solvents used in this study, twelve solvents interacted with PBTTT and six bad solvents were present inside the solubility window. Hence the Hildebrand theory wasn't able to provide a good estimate for the solubility window for PBTTT. Since the Hildebrand theory was not sufficient to explain the solubility data, the data was further analyzed using Hansen solubility theory. Figures 3B and 3C show the solubility data of PBTTT plotted using Hansen theory. The dispersion, polar and hydrogen bonding parameters of the solvents were plotted along the three axes. The 'good solvents' are plotted as filled circles and the 'bad solvents' are plotted as open circles. Computer software was used to fit a sphere that encompasses all the good solvents. Figure 3C show the two-dimensional representation of the Hansen plot for PBTTT. The center of sphere represents the Hansen parameters of the material. The dispersion, polar and hydrogen parameters for PBTTT are 17.30, 4.10, $4.00 \mathrm{MPa}^{0.5}$. The radius of interaction for PBTTT was 5.51. The accuracy of the solubility boundary depends on the number of bad solvents present inside the sphere. Out of thirty-three solvents, only two bad solvents were present inside the sphere. Hence, the Hansen solubility theory provided a better estimation of solubility boundary for PBTTT in comparison to Hildebrand solubility theory. 
The Hildebrand and Hansen solubility theory was also applied to a small molecule semiconductor (TIPS-pentacene). The solubility data for TIPS-pentacene is give in Table S1. Figure S1A-S1C shows the solubility data represented using Hildebrand and Hansen solubility theory. The Hansen theory was again able to provide an excellent fit for the solubility data. No bad solvents were present inside the solubility sphere of TIPS-pentacene.

\subsection{Solubility Theories Applied to Polymer Dielectrics}

The solubility of PMMA and PS in thirty-three selected solvents was studied. Solutions with concentration of $20 \mathrm{mg} / \mathrm{mL}$ were prepared. The solutions were heated overnight at $70^{\circ} \mathrm{C}$ to assist with the dissolution of the polymers. Solvents that dissolved the polymers completely were classified as 'good solvents' and the remaining solvents were classified as 'bad solvents'.

Table 2 shows the results from the solubility study for PMMA and PS. Figures 4A and $\mathbf{4 B}$ show the solubility data for PMMA and PS, respectively, represented using Hildebrand methodology. Solvents dissolving $20 \mathrm{mg} / \mathrm{mL}$ of PMMA and PS completely had a Hildebrand parameter value ranging from 17.4 26.7 and $17.4-21.7 \mathrm{MPa}^{0.5}$, respectively. The ester groups along the backbone of PMMA improves its solubility in organic solvents, leading to a larger solubility window as compared to PS. Similar to PBTTT, both PMMA and PS had many (six for PMMA and three for PS) non-solvents present inside their solubility window. In comparison, Hansen theory provided a better fit to the solubility data for PMMA and PS (Figure 4C - 4F). PS had one bad solvent present inside the Hansen sphere while PMMA had two bad solvents present inside the Hansen sphere. The dispersion, polar and hydrogen bonding parameters for PMMA and PS are 17.92, 7.34, 7.83 and $17.53,5.44,5.75 \mathrm{MPa}^{0.5}$, respectively. The radius of interaction for PMMA and PS was 9.42 and 5.56, respectively.

\subsection{Identification of Solvents Orthogonal to Organic Semiconductors}

The solubility study of the organic semiconductors and polymer dielectrics demonstrate that the Hansen solubility theory has a higher accuracy in defining solubility boundaries for a material in comparison to the Hildebrand theory. Once the Hansen solubility sphere for a material is built, the solubility or orthogonality of any organic solvent can be predicted to a good accuracy based on the location of the solvent with respect to the Hansen sphere. For the fabrication of top gate/bottom contact OFETs, we need to identify solvents that will dissolve the polymer gate dielectric but are orthogonal to the semiconductor. The Hansen solubility sphere for PBTTT, PMMA and PS were analyzed to identify solvents that dissolve the polymer dielectric but are orthogonal to the semiconductor. Out of thirty-three solvents, twenty-one solvents were orthogonal to PBTTT. Out of the twenty-one orthogonal solvents, six solvents dissolved PS and thirteen dissolved PMMA (Figure 5). Apart from the solubility of the polymer dielectric, printability of the dielectric solution on the semiconductor layer plays an important role. Printability is the ability of the solution to deposit a uniform layer of the material during the printing and drying process. The printability of a solvent depends on the surface energy of the substrate, and the surface tension and vapor pressure of the solvent. While considering printability of a solvent, the number of usable orthogonal solvents dropped to three and six for PS and PMMA, respectively (Figure 5).

In case of TIPS-pentacene, out of thirty-three solvents only five were orthogonal. Out of the five orthogonal solvents none dissolved PS, and only DMSO and methanol dissolved PMMA. Both DMSO and methanol based PMMA solutions had very poor printability, and the solutions dewet from the TIPSpentacene layer during the spin coating process. The Hansen solubility model predicts the interaction between the material and solvent but the printability of the solution cannot be predicted. 


\subsection{Top Gate/Bottom Contact PBTTT based OFETs}

Based on the analysis of the solubility data, we fabricated top gate/bottom contact PBTTT based OFETs with PMMA gate dielectric dissolved in solvents that are completely orthogonal to PBTTT and solvents that interact with PBTTT so some extent. PGMEA, propyl acetate, butyl acetate, ethyl acetate, dioxane and benzene were chosen as the solvent to dissolve PMMA. PGMEA, propyl acetate, butyl acetate and ethyl acetate were completely orthogonal towards PBTTT. Benzene (Grade C) and dioxane (Grade D) were non-orthogonal solvents and lay inside the Hansen sphere of PBTTT. Figures 6A - 6D show the location of the selected solvents in comparison to the Hansen sphere for PBTTT. The OFETs were fabricated using spin coating method on glass substrates with gold contacts treated with pentafluorobenzenethiol (PFBT) to increase their work function. ${ }^{46}$ The PBTTT layer was annealed at high temperature $\left(175^{\circ} \mathrm{C}\right)$ for 10 minutes to improve its molecular ordering. The output and transfer curves for the OFETs are presented in Figure 7 and $\mathbf{8}$. The mobility $\mu$ and threshold voltage $V_{T}$ were extracted from the standard equation in the saturation region,

$$
I_{D}=\left(\frac{W}{2 L}\right) \mu C_{i}\left(V_{G}-V_{T}\right)^{2}
$$

Where $I_{D}$ is the drain current, $W$ and $L$ are the channel width and length, respectively, $C_{i}$ is the capacitance per unit area of the gate dielectric, and $V_{G}$ is the gate voltage. The mobility of the devices under saturation regime ranged between 0.018 to $0.052 \mathrm{~cm}^{2} / \mathrm{Vs}$ (Table 3).

In this study, the concentration of the polymer dielectric solutions $(60 \mathrm{mg} / \mathrm{mL})$ and the spin speed (1500 RPM) was kept constant to remove the influence of viscosity and drying rate on morphology of the gate dielectric. Due to a large difference in the vapor pressure, boiling point, viscosity of the polymer dielectric solutions, and wettability of the dielectric solutions on PBTTT, the thickness of the dielectric layers after drying was in the range of 300 to $1500 \mathrm{~nm} .{ }^{1,7,47}$ The variation in dielectric thickness between the devices lead to differences in charge accumulation when the gate bias is kept at a constant voltage. To make a fair comparison between OFETs with different gate dielectric thicknesses, the mobilities at a constant gate bias of $0.25 \mathrm{MV} / \mathrm{cm}$ was calculated. By normalizing the data we observe that the mobility of devices processed with orthogonal solvents had a narrow distribution $\left(0.023\right.$ to $0.027 \mathrm{~cm}^{2} / \mathrm{Vs}$, Table 3$)$. The mobility of devices processed with dioxane (Grade-D solvent) was $0.024 \mathrm{~cm}^{2} / \mathrm{Vs}$, showing that grade-D solvent had very little influence on the morphology of PBTTT and they can be considered as orthogonal solvents due to their very poor interaction with the semiconductor at room temperature. The mobility of devices process with benzene (Grade-C solvent) was $0.018 \mathrm{~cm}^{2} / \mathrm{Vs}$ showing a slight influence due to swelling of the semiconductor. The electrical measurements show that PMMA polymer gate dielectric processed with orthogonal solvents has negligible effect on the electrical characteristics of the OFETs.

To investigate the effects of the polymer dielectric solution on the morphology of the semiconductor, the annealed PBTTT layers were immersed in PGMEA, propyl acetate, butyl acetate, ethyl acetate, dioxane and benzene for 2 minutes followed by spinning at 2000 RPM. The immersion process simulates the deposition of the dielectric solution on top of the semiconductor. Figure 9A and 9B shows the topographical AFM images of PBTTT layer, as spun and after annealing at $175^{\circ} \mathrm{C}$, respectively. The topographical AFM images show an increase in the domain size for the annealed sample as compared to the as spun sample. In general, the mobility and ON/OFF ratio of the OFETs improved after annealing the sample. ${ }^{7,48}$ Figures S2 and S3 show the amplitude and three-dimensional topographical AFM images of the samples. The amplitude images represented the slope of change in height. A rougher amplitude image indicates smaller domain size in comparison to a sample with smoother amplitude image. The amplitude image for the annealed sample (Figure S2B) is smoother as compared to the as spun sample (Figure S2A), confirming the increase in the domain size after the annealing process. The increase in roughness in the 
amplitude images (Figure S2C - S2H) for all samples after solvent treatment indicates that all solvents, both orthogonal and non-orthogonal, interact with PBTTT layer to some extent. In case of orthogonal solutions, the solvent reduces the average roughness $\left(\mathrm{R}_{\mathrm{a}}\right)$ of the PBTTT $(0.998$ to $1.079 \mathrm{~nm})$ in comparison to the sample with no solvent treatment $(1.097 \mathrm{~nm}$ ) (Figure 9). The samples treated with dioxane (Grade D) had a slightly higher surface roughness $\left(\mathrm{R}_{\mathrm{a}}\right), 1.114 \mathrm{~nm}$ but there was no significant difference in the mobility of devices as compared to devices processed with orthogonal solvents. Benzene (Grade $\mathrm{C}$ ) increased the surface roughness $\left(\mathrm{R}_{\mathrm{a}}\right)$ of PBTTT to $1.357 \mathrm{~nm}$ and a drop in the electrical mobility $\left(0.018 \mathrm{~cm}^{2} / \mathrm{Vs}\right)$ of the OFETs was observed. The non-orthogonal solvents interact with the semiconductor layer during the spin coating process, which swell the semiconductor layer and increase its surface roughness. ${ }^{49}$ Hence, nonorthogonal solvents are necessary to prevent interaction between the dielectric solution and the semiconductor layer.

\section{CONCLUSION}

Hansen theory had a higher accuracy in defining solubility boundaries for organic semiconductors and polymer dielectrics in comparison to Hildebrand theory. The Hansen solubility spheres for the organic semiconductors and polymer dielectrics can be used to identify solvents that will dissolve the polymer dielectric but have no interaction towards the semiconductor (orthogonal solvent) and vise-versa. A number of orthogonal dielectric solutions were identified for PBTTT by analyzing the solubility boundaries of PMMA and PBTTT. There was a negligible difference in mobility of OFETs processed with orthogonal dielectric solution whereas; the non-orthogonal dielectric solution swelled the semiconductor layer, which reduced the mobility of the OFETs. The methodology presented in this paper can be used for the fabrication of other solution processed multilayer structures where identification of good and orthogonal solvents is necessary. 


\section{AUTHOR INFORMATION}

\section{Corresponding Author}

* (A.M.G.) Email: agaikwad@ berkeley.edu

* (A.C.A.) Email: acarias@ eecs.berkeley.edu

\section{Present Addresses}

\#Electrical Engineering and Computer Sciences Department, University of California Berkeley, Cory Hall, Berkeley, California 94720, USA

${ }^{+}$Materials Sciences and Engineering Department, University of California Berkeley, Hearst Mining Building, Berkeley California 94720, USA.

\section{Author Contributions}

A.M.G. and A.C.A. conceived the project and designed the experiments. A.M.G, Y.K., A.E.O, S.P and S.A carried out the experiments. A.M.G and A.C.A wrote the paper and Y.K., A.E.O, S.P and S.A commented on the paper.

\section{Keywords}

Organic Semiconductors, Orthogonal Solvents, Hansen Solubility, TIPS-pentacene, PBTTT

\section{ACKNOWLEDGMENT}

The authors would like to thank Dr. Igal Deckman, Dr. Avinash Bhadani and Adrien Pierre for many helpful discussions and Prof. Lane Martin for access to the AFM instrument. This work is partially supported by the National Science Foundation under Grant No. EFRI 1240380. This work is also supported in part by Systems on Nanoscale Information fabriCs (SONIC), one of the six SRC STARnet Centers, sponsored by MARCO and DARPA. A.O. wishes to acknowledge financial support from the NSF Graduate Fellowship Research Program under Grant No. DGE-1106400. 


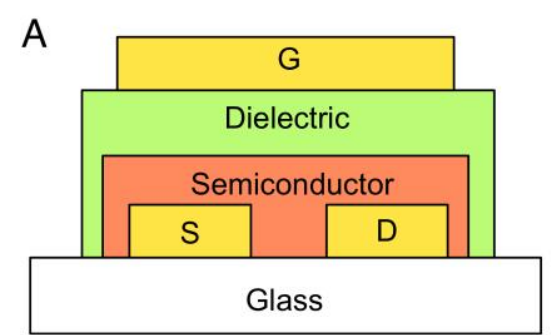

C

Dielectric

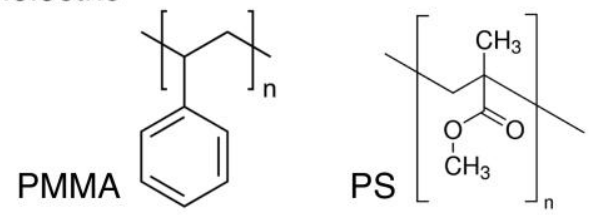

B

$\mathrm{D}$

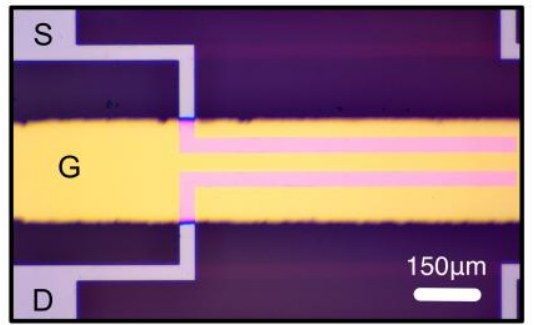

Semiconductor

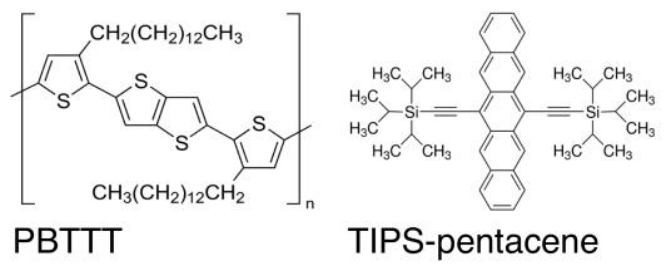

Figure 1. (A) Schematic representation and (B) optical micrograph of OFET with top gate/bottom contact architecture. (C) Chemical structure of polymer dielectrics (PMMA and PS) and (D) organic semiconductors (PBTTT and TIPS-pentacene). 


\begin{tabular}{|c|c|c|c|c|c|c|c|}
\hline \multirow[b]{2}{*}{ Solvent } & \multirow[b]{2}{*}{$\begin{array}{c}\mathrm{T} \\
\left(\mathrm{MPa}^{0.5}\right)\end{array}$} & \multirow[b]{2}{*}{$\begin{array}{c}\delta_{\mathrm{D}} \\
\left(\mathrm{MPa} a^{0.5}\right)\end{array}$} & \multirow[b]{2}{*}{$\begin{array}{c}\delta_{\mathrm{P}} \\
\left(\mathrm{MPa}^{0.5}\right)\end{array}$} & \multirow[b]{2}{*}{$\begin{array}{c}\delta_{\mathrm{H}} \\
\left(\mathrm{MPa}^{0.5}\right)\end{array}$} & \multicolumn{3}{|c|}{ РBTTT } \\
\hline & & & & & Sol. & RED & Grade \\
\hline 1-Butanol & 23.20 & 16.0 & 5.7 & 15.8 & 1 & 2.2 & $\mathrm{D}$ \\
\hline Dioxane & 20.47 & 19.0 & 1.8 & 7.4 & 1 & 0.9 & $\mathrm{D}$ \\
\hline 2-Methoxyethanol & 24.82 & 16.2 & 9.2 & 16.4 & 0 & 2.5 & $E$ \\
\hline Acetone & 19.94 & 15.5 & 10.4 & 7.0 & 0 & 1.4 & $E$ \\
\hline Acetophenone & 21.72 & 19.6 & 8.6 & 3.7 & 0 & 1.1 & $E$ \\
\hline Benzene & 18.51 & 18.4 & 0.0 & 2.0 & 1 & 0.9 & $\mathrm{C}$ \\
\hline Benzyl alcohol & 23.79 & 18.4 & 6.3 & 13.7 & 0 & 1.8 & $E$ \\
\hline Butyl acetate & 17.41 & 15.8 & 3.7 & 6.3 & 0 & 0.8 & $E$ \\
\hline Carbon tetrachloride & 17.81 & 17.8 & 0.0 & 0.6 & 1 & 1.0 & C \\
\hline Chlorobenzene & 19.58 & 19.0 & 4.3 & 2.0 & 1 & 0.6 & $A$ \\
\hline Chloroform & 18.95 & 17.8 & 3.1 & 5.7 & 1 & 0.4 & $A$ \\
\hline Cyclohexanol & 22.40 & 17.4 & 4.1 & 13.5 & 0 & 1.7 & $E$ \\
\hline Cyclopentanone & 17.90 & 16.7 & 3.8 & 5.2 & 1 & 0.4 & $\mathrm{D}$ \\
\hline Dichlorobenzene & 19.87 & 18.6 & 6.2 & 3.2 & 1 & 0.5 & $A$ \\
\hline Dietheyl carbonate & 17.63 & 16.3 & 3.0 & 6.0 & 0 & 0.6 & $E$ \\
\hline Dimethyl formamide & 24.86 & 17.4 & 13.7 & 11.3 & 0 & 1.2 & $E$ \\
\hline Dimethyl sulfoxide & 26.68 & 18.4 & 16.4 & 10.2 & 0 & 2.1 & $E$ \\
\hline Ethanol & 26.52 & 15.8 & 8.8 & 19.4 & 0 & 2.5 & $E$ \\
\hline Ethoxyethanol & 22.95 & 15.8 & 9.0 & 14.0 & 0 & 3.0 & $E$ \\
\hline Ethyl acetate & 18.15 & 15.8 & 5.3 & 7.2 & 0 & 2.1 & $E$ \\
\hline Ethylene dichloride & 20.80 & 19.0 & 7.4 & 4.1 & 0 & 0.9 & $E$ \\
\hline Ethylene glycol & 32.95 & 17.0 & 11.0 & 26.0 & 0 & 4.2 & $E$ \\
\hline Formamide & 36.65 & 17.2 & 26.2 & 19.0 & 0 & 4.8 & $E$ \\
\hline Hexane & 14.90 & 14.9 & 0.0 & 0.0 & 0 & 1.4 & $E$ \\
\hline Isophorone & 19.94 & 16.6 & 8.2 & 7.4 & 1 & 1.0 & D \\
\hline Mesitylene & 18.01 & 18.0 & 0.0 & 0.6 & 1 & 1.0 & B \\
\hline Methanol & 29.61 & 15.1 & 12.3 & 22.3 & 0 & 3.7 & $E$ \\
\hline Propyl acetate & 17.62 & 15.3 & 4.3 & 7.6 & 0 & 1.1 & $E$ \\
\hline Propylene glycol & 30.22 & 16.8 & 9.4 & 23.3 & 0 & 3.6 & $E$ \\
\hline PGMEA & 19.26 & 15.6 & 5.6 & 9.8 & 0 & 1.3 & $E$ \\
\hline Tetrahydrofuran & 19.46 & 16.8 & 5.7 & 8.0 & 1 & 0.8 & B \\
\hline Toluene & 18.16 & 18.0 & 1.4 & 2.0 & 1 & 0.6 & B \\
\hline Tetralin & 19.01 & 18.7 & 2.0 & 2.8 & 1 & 1.0 & $A$ \\
\hline
\end{tabular}

Table 1. List of solvents used for the solubility study of PBTTT and their Hansen parameters. The good solvents are marked as ' 1 ' and the bad solvents are marked as ' 0 '. RED number (Relative Energy Difference) gives the distance of the solvent relative to the radius of the Hansen sphere for the organic semiconductors. The grade represents the degree of solubility of the solvents. Grade-A solvents dissolve the organic semiconductor completely, while grade-E solvents are completely orthogonal to the semiconductor. 


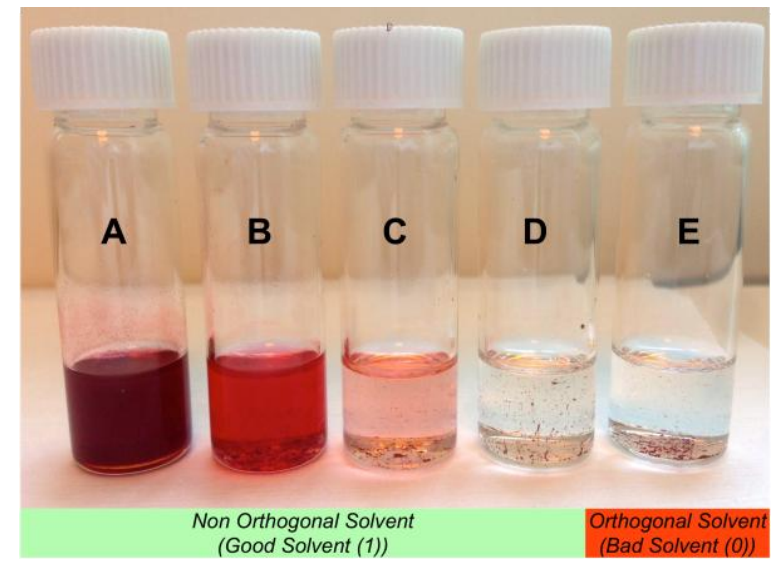

Figure 2. Supernatant of grade A, B, C, D and E solvents used to dissolve PBTTT. 
A
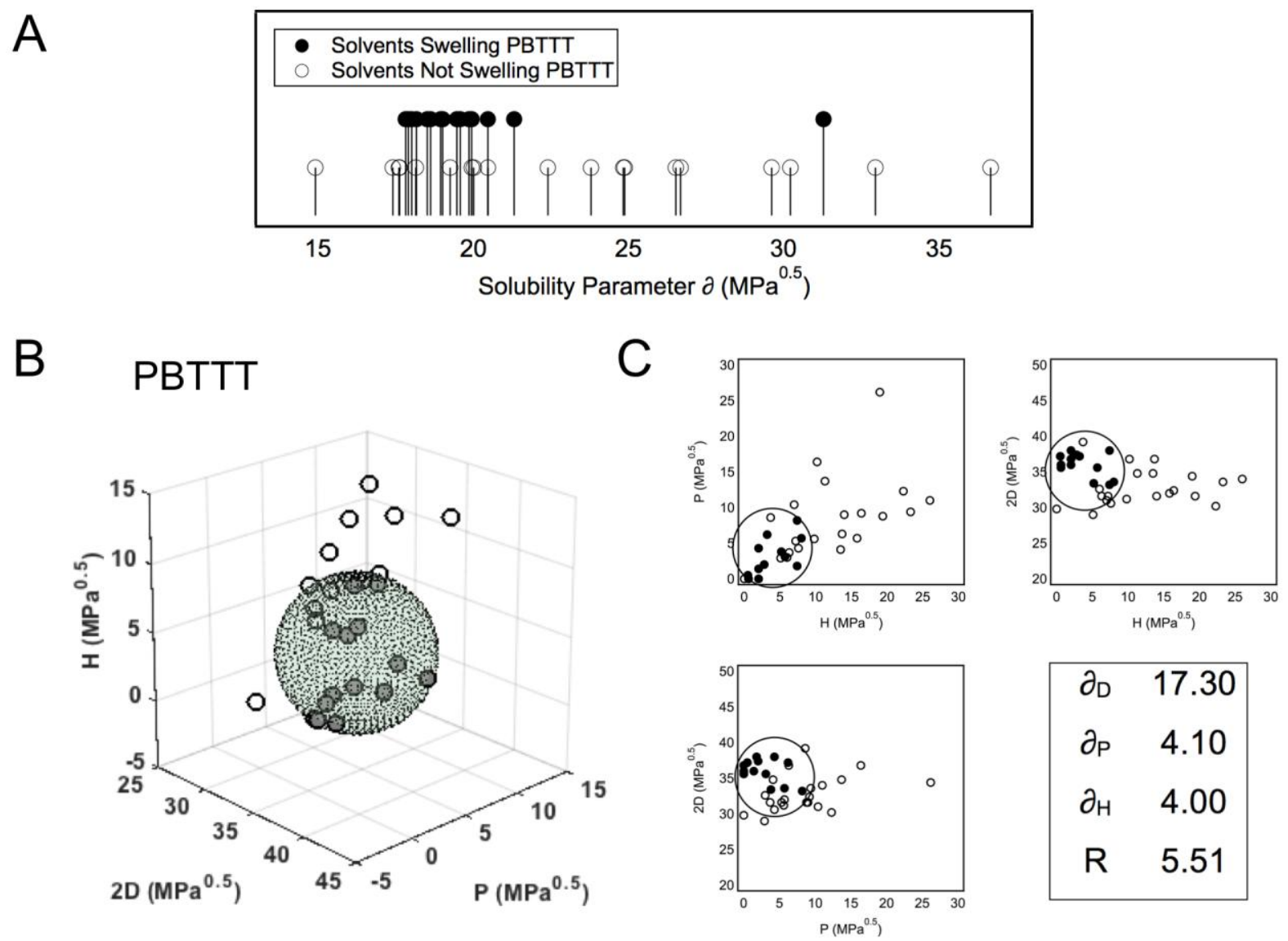

$\begin{array}{cc}\partial_{D} & 17.30 \\ \partial_{P} & 4.10 \\ \partial_{H} & 4.00 \\ R & 5.51\end{array}$

Figure 3. (A) Solubility data of PBTTT represented using Hildebrand theory. (B) Three-dimensional representation of the solubility data of PBTTT using Hansen theory. (C) Two-dimensional Hansen plot and the Hansen parameters for PBTTT. 


\begin{tabular}{|c|c|c|c|c|c|c|c|c|}
\hline \multirow[b]{2}{*}{ Solvent } & \multirow[b]{2}{*}{$\begin{array}{c}\mathrm{T}^{\mathrm{T}} \\
\left(\mathrm{MPa}^{0.5}\right)\end{array}$} & \multirow[b]{2}{*}{$\begin{array}{c}\delta_{\mathrm{D}} \\
\left(\mathrm{MPa}^{0.5}\right)\end{array}$} & \multirow[b]{2}{*}{$\begin{array}{c}\delta_{\mathrm{P}} \\
\left(\mathrm{MPa}^{0.5}\right)\end{array}$} & \multirow[b]{2}{*}{$\begin{array}{c}\delta_{\mathrm{H}} \\
\left(\mathrm{MPa}^{0.5}\right)\end{array}$} & \multicolumn{2}{|c|}{ PMMA } & \multicolumn{2}{|c|}{ PS } \\
\hline & & & & & Solubilty & RED & Solubilty & RED \\
\hline 1-Butanol & 23.20 & 16.0 & 5.7 & 15.8 & 0 & 1.0 & 0 & 1.9 \\
\hline Dioxane & 20.47 & 19.0 & 1.8 & 7.4 & 1 & 0.6 & 1 & 0.9 \\
\hline 2-Methoxyethanol & 24.82 & 16.2 & 9.2 & 16.4 & 1 & 1.0 & 0 & 2.1 \\
\hline Acetone & 19.94 & 15.5 & 10.4 & 7.0 & 1 & 0.6 & 0 & 1.2 \\
\hline Acetophenone & 21.72 & 19.6 & 8.6 & 3.7 & 1 & 0.6 & 1 & 1.0 \\
\hline Benzene & 18.51 & 18.4 & 0.0 & 2.0 & 1 & 1.0 & 0 & 1.2 \\
\hline Benzyl alcohol & 23.79 & 18.4 & 6.3 & 13.7 & 1 & 0.6 & 0 & 1.5 \\
\hline Butyl acetate & 17.41 & 15.8 & 3.7 & 6.3 & 1 & 0.6 & 1 & 0.7 \\
\hline Carbon tetrachloride & 17.81 & 17.8 & 0.0 & 0.6 & 0 & 1.1 & 0 & 1.3 \\
\hline Chlorobenzene & 19.58 & 19.0 & 4.3 & 2.0 & 1 & 0.7 & 1 & 0.9 \\
\hline Chloroform & 18.95 & 17.8 & 3.1 & 5.7 & 1 & 0.5 & 1 & 0.4 \\
\hline Cyclohexanol & 22.40 & 17.4 & 4.1 & 13.5 & 0 & 0.7 & 0 & 1.4 \\
\hline Cyclopentanone & 17.90 & 16.7 & 3.8 & 5.2 & 1 & 0.5 & 1 & 0.4 \\
\hline Dichlorobenzene & 19.87 & 18.6 & 6.2 & 3.2 & 1 & 0.5 & 1 & 0.6 \\
\hline Dietheyl carbonate & 17.63 & 16.3 & 3.0 & 6.0 & 1 & 0.6 & 1 & 0.6 \\
\hline Dimethyl formamide & 24.86 & 17.4 & 13.7 & 11.3 & 1 & 0.8 & 0 & 1.2 \\
\hline Dimethyl sulfoxide & 26.68 & 18.4 & 16.4 & 10.2 & 1 & 1.0 & 0 & 1.8 \\
\hline Ethanol & 26.52 & 15.8 & 8.8 & 19.4 & 0 & 1.3 & 0 & 2.1 \\
\hline Ethoxyethanol & 22.95 & 15.8 & 9.0 & 14.0 & 1 & 0.8 & 0 & 2.6 \\
\hline Ethyl acetate & 18.15 & 15.8 & 5.3 & 7.2 & 1 & 0.5 & 0 & 1.7 \\
\hline Ethylene dichloride & 20.80 & 19.0 & 7.4 & 4.1 & 1 & 0.5 & 1 & 0.7 \\
\hline Ethylene glycol & 32.95 & 17.0 & 11.0 & 26.0 & 0 & 2.0 & 0 & 3.8 \\
\hline Formamide & 36.65 & 17.2 & 26.2 & 19.0 & 0 & 2.3 & 0 & 4.4 \\
\hline Hexane & 14.90 & 14.9 & 0.0 & 0.0 & 0 & 1.3 & 0 & 1.7 \\
\hline Isophorone & 19.94 & 16.6 & 8.2 & 7.4 & 0 & 0.3 & 1 & 0.7 \\
\hline Mesitylene & 18.01 & 18.0 & 0.0 & 0.6 & 0 & 1.1 & 0 & 1.4 \\
\hline Methanol & 29.61 & 15.1 & 12.3 & 22.3 & 0 & 1.7 & 0 & 3.3 \\
\hline Propyl acetate & 17.62 & 15.3 & 4.3 & 7.6 & 1 & 0.6 & 1 & 0.9 \\
\hline Propylene glycol & 30.22 & 16.8 & 9.4 & 23.3 & 0 & 1.7 & 0 & 3.2 \\
\hline PGMEA & 19.26 & 15.6 & 5.6 & 9.8 & 1 & 0.6 & 1 & 1.0 \\
\hline Tetrahydrofuran & 19.46 & 16.8 & 5.7 & 8.0 & 1 & 0.3 & 1 & 0.5 \\
\hline Toluene & 18.16 & 18.0 & 1.4 & 2.0 & 1 & 0.9 & 1 & 1.0 \\
\hline Tetralin & 19.01 & 18.7 & 2.0 & 2.8 & 1 & 0.8 & 1 & 0.9 \\
\hline
\end{tabular}

Table 2. List of solvents used for the solubility study of PMMA and PS, and their Hansen parameters. The good solvents are marked as ' 1 ' and the bad solvents are marked as ' 0 '. RED number (Relative Energy Difference) gives the distance of the solvent relative to the radius of the Hansen sphere for the polymers. 

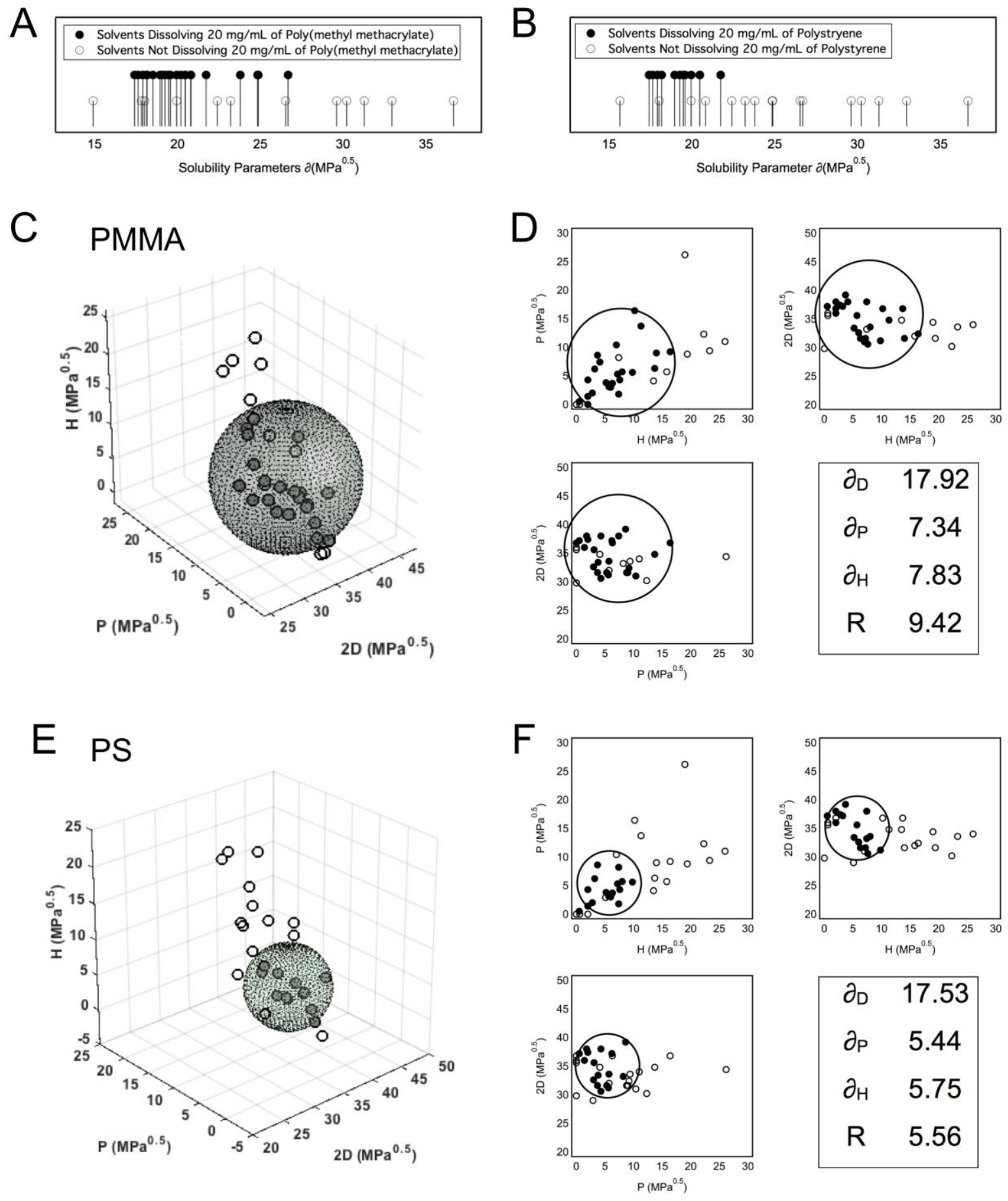

\begin{tabular}{|ll|}
\hline$\partial_{D}$ & 17.53 \\
$\partial_{P}$ & 5.44 \\
$\partial_{H}$ & 5.75 \\
$R$ & 5.56 \\
\hline
\end{tabular}

Figure 4. Solubility data of PMMA (A) and PS (B) represented using Hildebrand theory. Three-dimensional representation of solubility data of PMMA (C) and PS (E) using Hansen theory. (D) And (F) show the twodimensional Hansen plot and the Hansen parameters for PMMA and PS, respectively. 


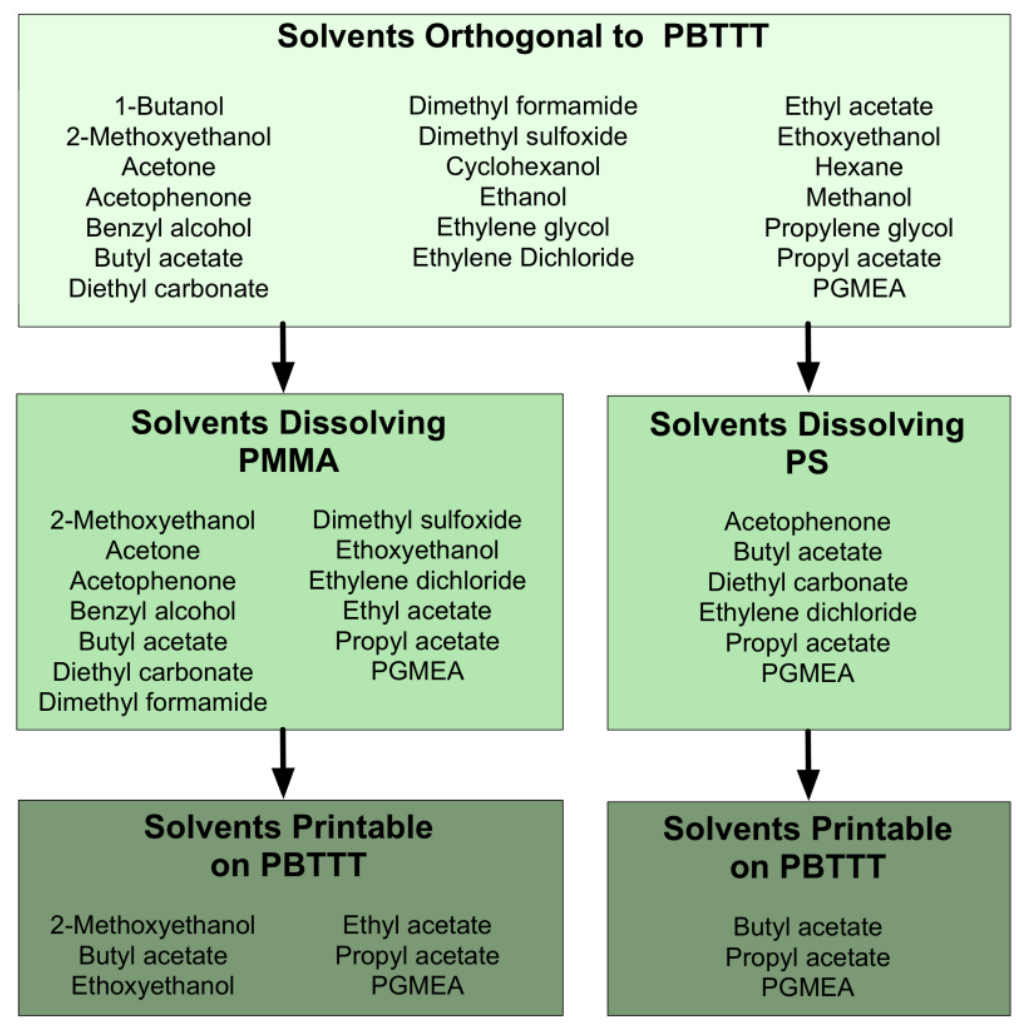

Figure 5. Schematic summarizing the solvents orthogonal to PBTTT, the solubility of PMMA and PS in the orthogonal solvents, and their printabiltiy on PBTTT. 

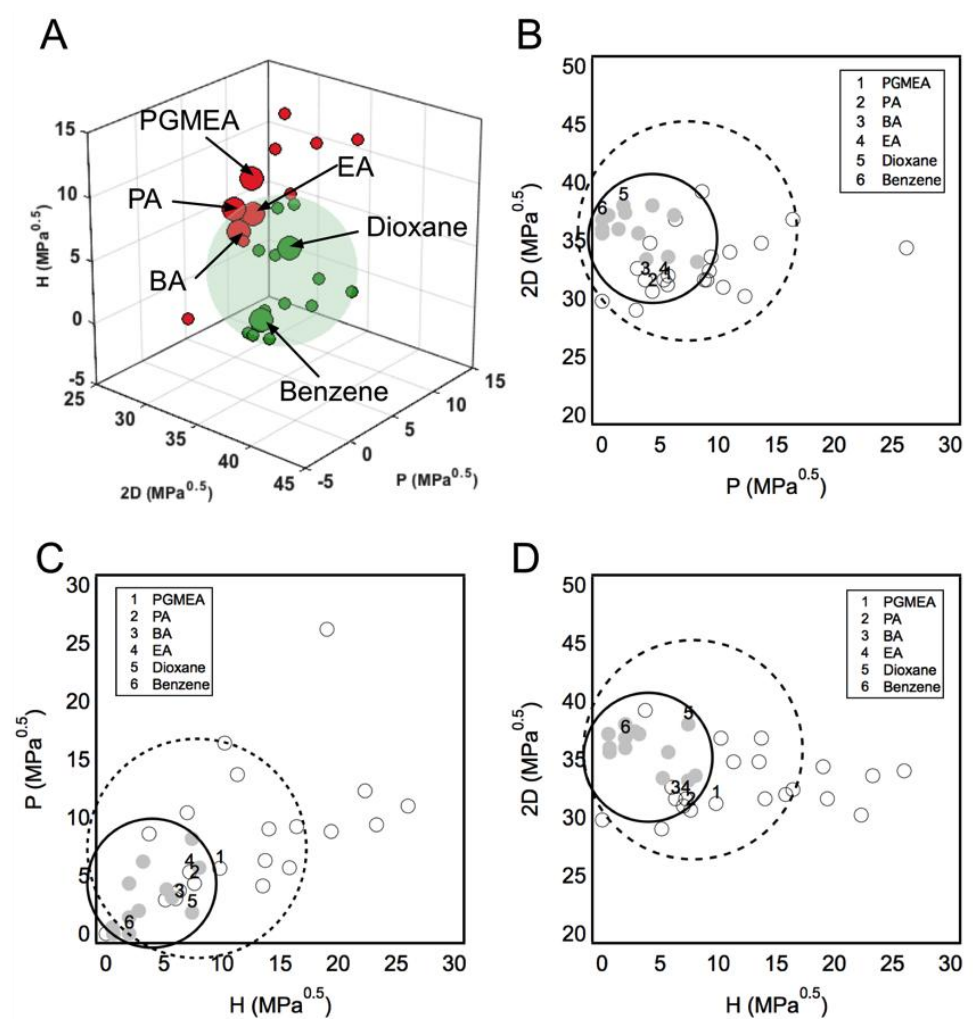

Figure 6. (A) Location of PGMEA, EA, PA, BA, dioxane and benzene in the Hansen space. The green sphere represents the Hansen solubiltiy sphere for PBTTT. (B, C, D) Two dimensional Hansen plot for PBTTT. The gray solid and open circles represents good and bad solvents for PBTTT, respectively. The solid and dash circles represent the Hansen solubility sphere for PBTTT and PMMA, respectively. 

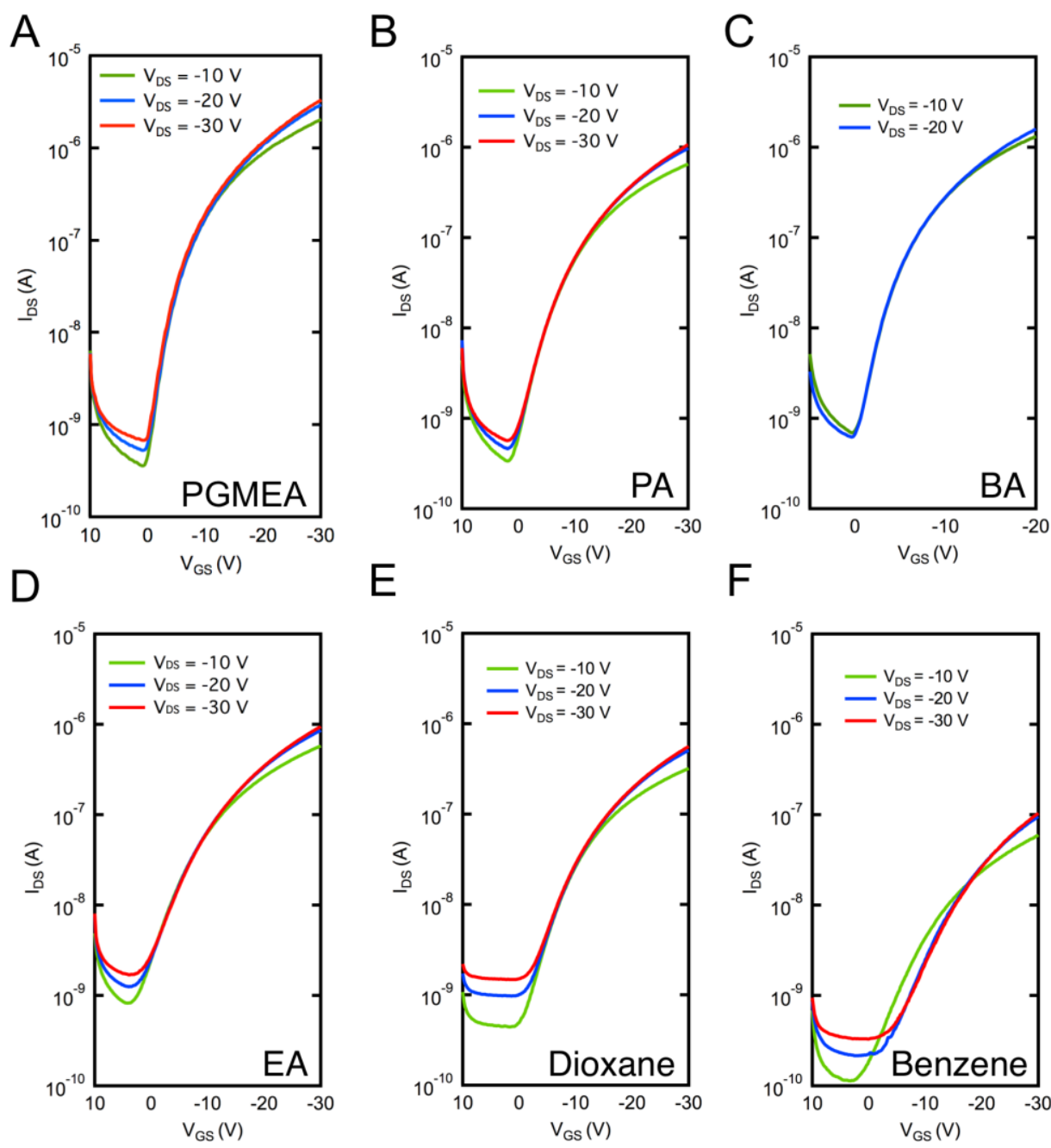

\section{$\mathrm{F}$}

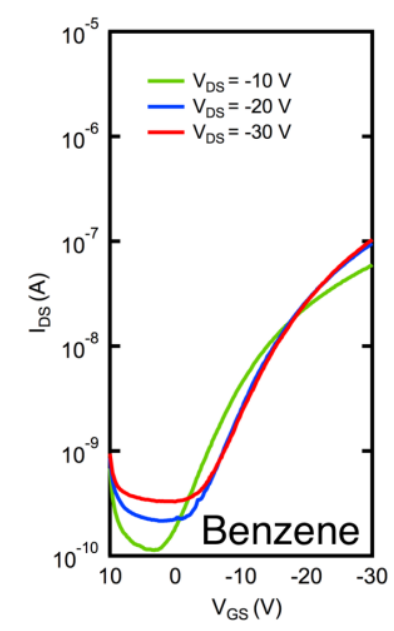

Figure 7. Transfer characteristics of top gate/bottom contact PBTTT based OFETs with PMMA gate dielectric using (a) PGMEA, (b) Propyl acetate, (c) Butyl acetate, (d) Ethyl acetate, (e) Dioxane and (f) Benzene as the solvent, respectively. 
A
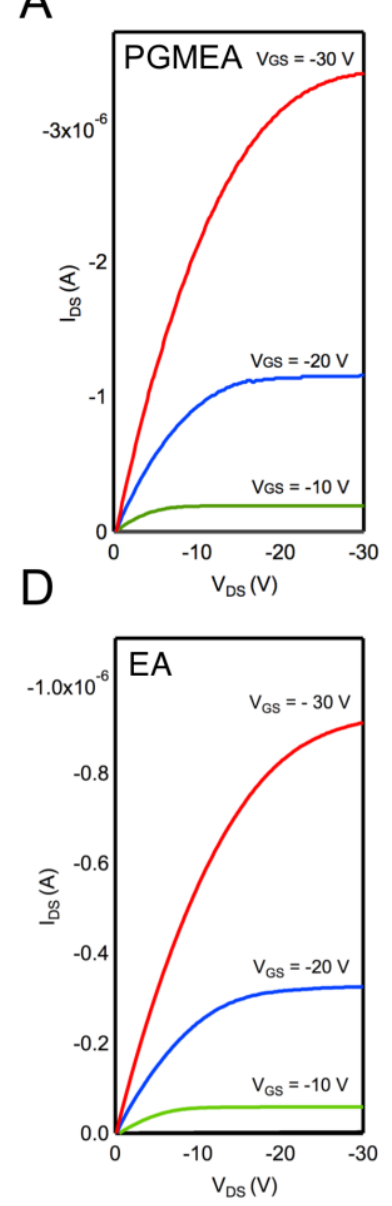

$B$

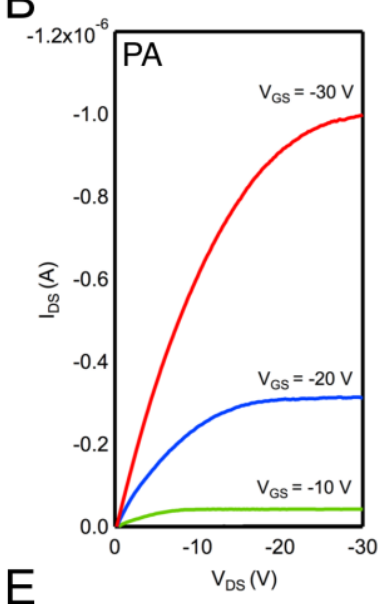

$\mathrm{E}$

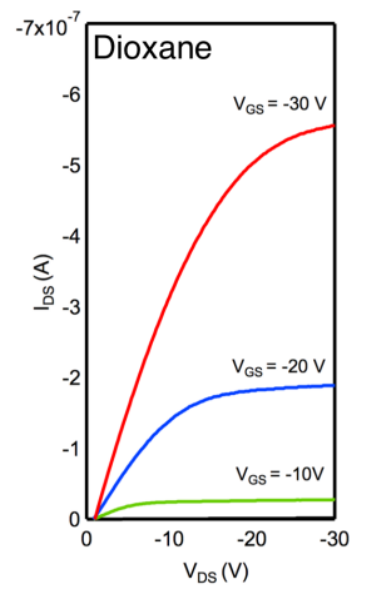

C

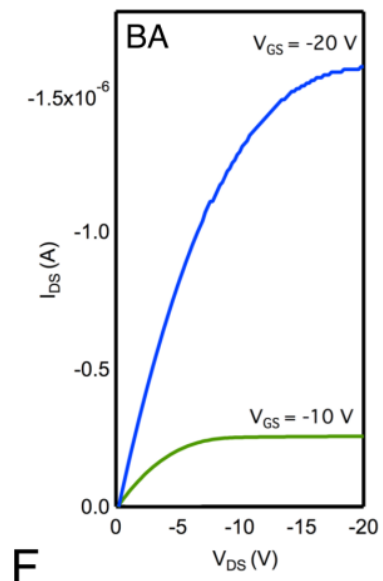

$\mathrm{F}$

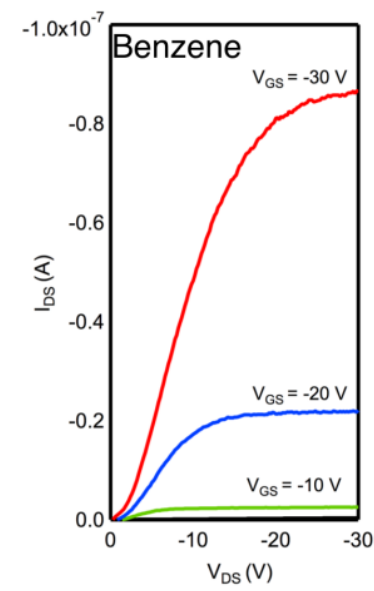

Figure 8. Output characteristics of top gate/bottom contact PBTTT based OFETs with PMMA gate dielectric using (a) PGMEA, (b) Propyl acetate, (c) Butyl acetate, (d) Ethyl acetate, (e) Dioxane and (f) Benzene as the solvent, respectively. 


\begin{tabular}{|c|c|c|c|c|c|c|c|c|}
\hline Solvent & $\begin{array}{l}\text { Thickness } \\
\text { (nm) }\end{array}$ & $\begin{array}{l}\text { Mobility } \\
\left(\mathrm{cm}^{2} / \mathrm{Vs}\right)\end{array}$ & Threshold (V) & ON/OFF & SS (V/dec) & $\begin{array}{l}\text { Solubiltiy } \\
\text { Rating }\end{array}$ & $\begin{array}{c}\mathrm{R}_{\mathrm{a}} \\
(\mathrm{nm})\end{array}$ & $\frac{\frac{\text { Mobility at }}{0.25 \mathrm{MV} / \mathrm{cm}^{c}}}{\left(\mathrm{~cm}^{2} / \mathrm{Vs}\right)}$ \\
\hline PGMEA & 300 & $0.052( \pm 0.002)$ & $-5.56( \pm 0.16)$ & $\sim 10^{3.8}$ & $3.11( \pm 0.03)$ & $E$ & 1.014 & $\underline{0.026( \pm 0.001)}$ \\
\hline PA & 490 & $0.041( \pm 0.003)$ & $-7.72( \pm 0.63)$ & $\sim 10^{3.7}$ & $5.29( \pm 0.94)$ & $E$ & 0.998 & $\underline{0.025( \pm 0.002)}$ \\
\hline$B A^{b}$ & 180 & $0.036( \pm 0.002)$ & $-3.93( \pm 0.21)$ & $\sim 10^{3.6}$ & $2.79( \pm 0.43)$ & E & 1.079 & $\underline{0.023( \pm 0.002)}$ \\
\hline EA & 760 & $0.039( \pm 0.002)$ & $-7.37( \pm 0.95)$ & $\sim 10^{3.2}$ & $7.14( \pm 0.34)$ & $E$ & 1.066 & $\underline{0.027( \pm 0.001)}$ \\
\hline Dioxane & 683 & $0.030( \pm 0.002)$ & $-6.94( \pm 0.55)$ & $\sim 10^{3.0}$ & $6.70( \pm 0.28)$ & $\mathrm{D}$ & 1.114 & $\underline{0.024( \pm 0.002)}$ \\
\hline Benzene & 1524 & $0.018( \pm 0.002)$ & $-13.04( \pm 1.95)$ & $\sim 10^{3.0}$ & $10.73( \pm 2.66)$ & C & 1.357 & $\underline{0.018( \pm 0.002)}$ \\
\hline
\end{tabular}

a) The mobility was measured in the saturation regime at $V_{G S}=V_{D S}=30 \mathrm{~V}$.

b) The mobility of devices with $B A$ as the solvent for PMMA was measured at $V_{G S}=V_{D S}=20 \mathrm{~V}$.

c) The mobility was measured at gate bias of $0.25 \mathrm{MV} / \mathrm{cm}$.

Table 3. Electrical parameters of PBTTT based OFETs with top gate/ bottom contact architecture $(\mathrm{W} / \mathrm{L}=$ $1 \mathrm{~mm} / 50 \mu \mathrm{m})$. The field-effect mobilities were calculated in the saturated regime using standard device formalism. 

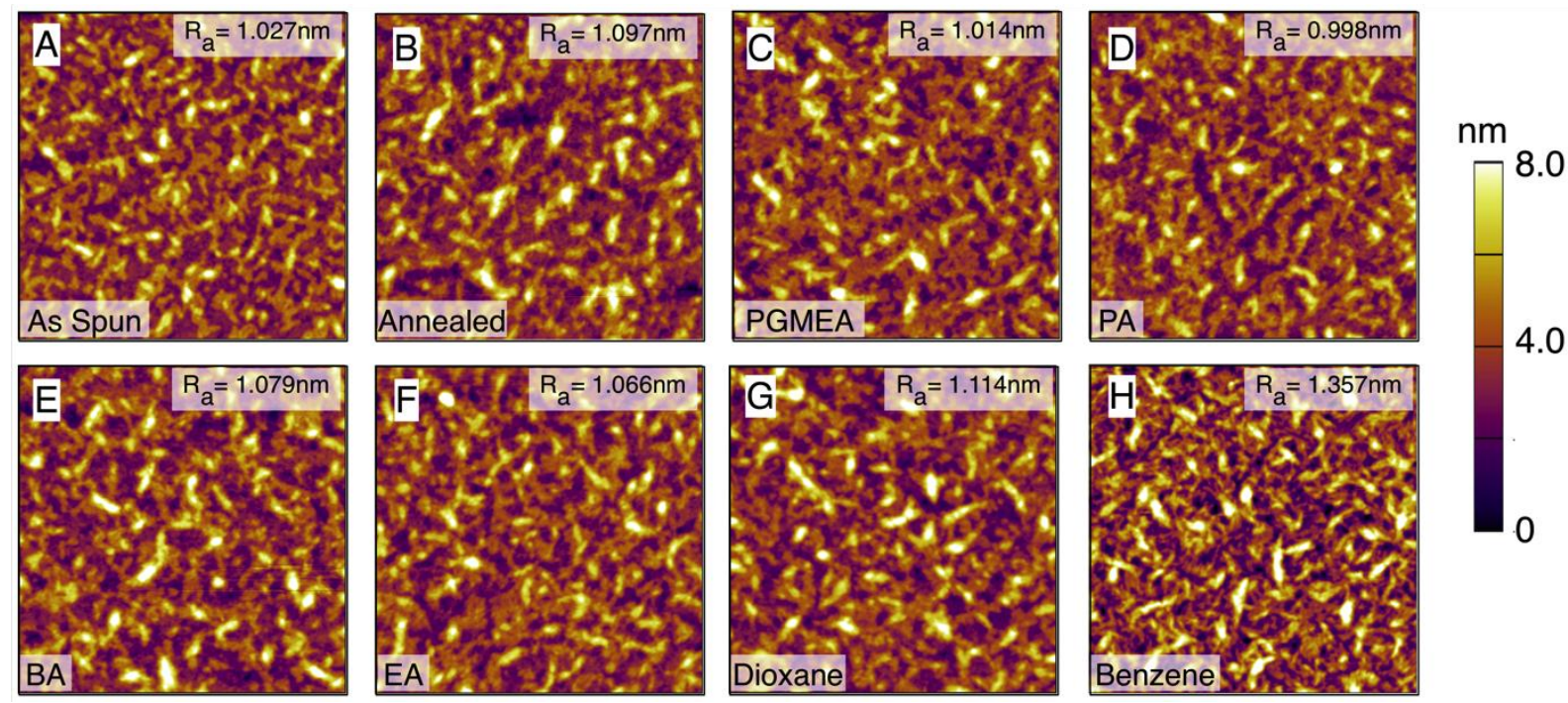

Figure 9. Topographical atomic force microscopy (AFM) images $(2 \mu \mathrm{m} \times 2 \mu \mathrm{m})$ of spin coated PBTTT film as spun (A) and after annealing at $175^{\circ} \mathrm{C}$ for $10 \mathrm{~min}(\mathrm{~B})$, and annealed PBTTT film after immersing in PGMEA (C), propyl acetate (D), butyl acetate (E), ethyl acetate (F), dioxane $(\mathrm{G})$ and benzene $(\mathrm{H})$ for 2 minutes followed by spinning at $2000 \mathrm{RPM}$ and annealing at $110^{\circ} \mathrm{C}$ for $1 \mathrm{hr}$. 


\section{REFERENCES}

(1) De La Fuente Vornbrock, A.; Sung, D.; Kang, H.; Kitsomboonloha, R.; Subramanian, V. Fully Gravure and Ink-Jet Printed High Speed pBTTT Organic Thin Film Transistors. Org. Electron. 2010, 11, 2037-2044.

(2) Sirringhaus, H.; Kawase, T.; Friend, R. H.; Shimoda, T.; Inbasekaran, M.; Wu, W.; Woo, E. P. HighResolution Inkjet Printing of All-Polymer Transistor Circuits. Science 2000, 290, 2123-2126.

(3) Kempa, H.; Hambsch, M.; Reuter, K.; Stanel, M.; Schmidt, G. C.; Meier, B.; Hübler, A. C. Complementary Ring Oscillator Exclusively Prepared by Means of Gravure and Flexographic Printing. IEEE Trans. Electron Devices 2011, 58, 2765-2769.

(4) Søndergaard, R. R.; Hösel, M.; Krebs, F. C. Roll-to-Roll Fabrication of Large Area Functional Organic Materials. J. Polym. Sci. Part B Polym. Phys. 2013, 51, 16-34.

(5) Kang, B.; Lee, W. H.; Cho, K. Recent Advances in Organic Transistor Printing Processes. ACS Appl. Mater. Interfaces 2013, 5, 2302-2315.

(6) Yan, H.; Chen, Z.; Zheng, Y.; Newman, C.; Quinn, J. R.; Dötz, F.; Kastler, M.; Facchetti, A. A HighMobility Electron-Transporting Polymer for Printed Transistors. Nature 2009, 457, 679-686.

(7) McCulloch, I.; Heeney, M.; Bailey, C.; Genevicius, K.; Macdonald, I.; Shkunov, M.; Sparrowe, D.; Tierney, S.; Wagner, R.; Zhang, W.; et al. Liquid-Crystalline Semiconducting Polymers with High Charge-Carrier Mobility. Nat. Mater. 2006, 5, 328-333.

(8) Mei, Y.; Loth, M. A.; Payne, M.; Zhang, W.; Smith, J.; Day, C. S.; Parkin, S. R.; Heeney, M.; McCulloch, I.; Anthopoulos, T. D.; et al. High Mobility Field-Effect Transistors with Versatile Processing from a Small-Molecule Organic Semiconductor. Adv. Mater. 2013, 25, 4352-4357.

(9) Park, Y. D.; Park, Y. D.; Lim, J. A.; Lim, J. A.; Lee, H. S.; Lee, H. S.; Cho, K.; Cho, K. Interface Engineering in Organic Transistors. Mater. Today 2007, 10, 46-54.

(10) Diao, Y.; Tee, B. C.-K.; Giri, G.; Xu, J.; Kim, D. H.; Becerril, H. A.; Stoltenberg, R. M.; Lee, T. H.; Xue, G.; Mannsfeld, S. C. B.; et al. Solution Coating of Large-Area Organic Semiconductor Thin Films with Aligned Single-Crystalline Domains. Nat. Mater. 2013, 12, 665-671.

(11) Gundlach, D. J.; Jackson, T. N.; Schlom, D. G.; Nelson, S. F. Solvent-Induced Phase Transition in Thermally Evaporated Pentacene Films. Appl. Phys. Lett. 1999, 74, 3302-3304.

(12) Sirringhaus, H. 25th Anniversary Article: Organic Field-Effect Transistors: The Path beyond Amorphous Silicon. Adv. Mater. 2014, 26, 1319-1335.

(13) Sirringhaus, H. Reliability of Organic Field-Effect Transistors. Adv. Mater. 2009, 21, 3859-3873.

(14) Street, R. A.; Nga Ng, T.; Schwartz, D. E.; Whiting, G. L.; Lu, J. P.; Bringans, R. D.; Veres, J. From Printed Transistors to Printed Smart Systems. Proc. IEEE 2015, 103.

(15) Arias, A. C.; MacKenzie, J. D.; McCulloch, I.; Rivnay, J.; Salleo, A. Materials and Applications for Large Area Electronics: Solution-Based Approaches. Chem. Rev. 2010, 110, 3-24. 
(16) Loo, Y.-L.; McCulloch, I. Progress and Challenges in Commercialization of Organic Electronics. MRS Bull. 2008, 33, 653-662.

(17) Ng, T. N.; Wong, W. S.; Chabinyc, M. L.; Sambandan, S.; Street, R. a. Flexible Image Sensor Array with Bulk Heterojunction Organic Photodiode. Appl. Phys. Lett. 2008, 92, 2013-2016.

(18) Klauk, H. Organic Thin-Film Transistors. Chem. Soc. Rev. 2010, 39, 2643-2666.

(19) Veres, J.; Ogier, S.; Lloyd, G. Gate Insulators in Organic Field-Effect Transistors. Chem. Mater. 2004, 4543-4555.

(20) Park, S. K.; Jackson, T. N.; Anthony, J. E.; Mourey, D. A. High Mobility Solution Processed 6,13Bis(triisopropyl-Silylethynyl) Pentacene Organic Thin Film Transistors. Appl. Phys. Lett. 2007, 91, 34-37.

(21) Facchetti, A.; Yoon, M.-H.; Marks, T. J. Gate Dielectrics for Organic Field-Effect Transistors: New Opportunities for Organic Electronics. Adv. Mater. 2005, 17, 1705-1725.

(22) Yoon, M. H.; Yan, H.; Facchetti, A.; Marks, T. J. Low-Voltage Organic Field-Effect Transistors and Inverters Enabled by Ultrathin Cross-Linked Polymers as Gate Dielectrics. J. Am. Chem. Soc. 2005, $127,10388-10395$.

(23) Chua, L.-L.; Zaumseil, J.; Chang, J.-F.; Ou, E. C.-W.; Ho, P. K.-H.; Sirringhaus, H.; Friend, R. H. General Observation of N-Type Field-Effect Behaviour in Organic Semiconductors. Nature 2005, 434, 194-199.

(24) Hamadani, B. H.; Gundlach, D. J.; McCulloch, I.; Heeney, M. Undoped Polythiophene Field-Effect Transistors with Mobility of 1 cm2 V-1 S-1. Appl. Phys. Lett. 2007, 91, 2005-2008.

(25) Lee, S.; Koo, B.; Shin, J.; Lee, E.; Park, H.; Kim, H. Effects of Hydroxyl Groups in Polymeric Dielectrics on Organic Transistor Performance. Appl. Phys. Lett. 2006, 88, 2004-2007.

(26) Liu, C.; Xu, Y.; Noh, Y.-Y. Contact Engineering in Organic Field-Effect Transistors. Mater. Today 2015, 18, 79-96.

(27) Natali, D.; Caironi, M. Charge Injection in Solution-Processed Organic Field-Effect Transistors: Physics, Models and Characterization Methods. Adv. Mater. 2012, 24, 1357-1387.

(28) Richards, T. J.; Sirringhaus, H. Analysis of the Contact Resistance in Staggered, Top-Gate Organic Field-Effect Transistors. J. Appl. Phys. 2007, 102.

(29) Gundlach, D. J.; Zhou, L.; Nichols, J. A.; Jackson, T. N.; Necliudov, P. V.; Shur, M. S. An Experimental Study of Contact Effects in Organic Thin Film Transistors. J. Appl. Phys. 2006, 100.

(30) Hill, I. G. Numerical Simulations of Contact Resistance in Organic Thin-Film Transistors. Appl. Phys. Lett. 2005, 87, 1-3.

(31) Baeg, K.-J.; Facchetti, A.; Noh, Y.-Y. Effects of Gate Dielectrics and Their Solvents on Characteristics of Solution-Processed N-Channel Polymer Field-Effect Transistors. J. Mater. Chem. 2012, 22, 21138. 
(32) Klauk, H.; Halik, M.; Zschieschang, U.; Schmid, G.; Radlik, W.; Weber, W. High-Mobility Polymer Gate Dielectric Pentacene Thin Film Transistors. J. Appl. Phys. 2002, 92, 5259-5263.

(33) Yang, S. Y.; Kim, S. H.; Shin, K.; Jeon, H.; Park, C. E. Low-Voltage Pentacene Field-Effect Transistors with Ultrathin Polymer Gate Dielectrics. Appl. Phys. Lett. 2006, 88, 3-5.

(34) Chua, L. L.; Ho, P. K. H.; Sirringhaus, H.; Friend, R. H. High-Stability Ultrathin Spin-on Benzocyclobutene Gate Dielectric for Polymer Field-Effect Transistors. Appl. Phys. Lett. 2004, 84, 3400-3402.

(35) Zakhidov, A. A.; Lee, J.-K.; DeFranco, J. A.; Fong, H. H.; Taylor, P. G.; Chatzichristidi, M.; Ober, C. K.; Malliaras, G. G. Orthogonal Processing: A New Strategy for Organic Electronics. Chem. Sci. 2011, 2, 1178.

(36) MacHui, F.; Abbott, S.; Waller, D.; Koppe, M.; Brabec, C. J. Determination of Solubility Parameters for Organic Semiconductor Formulations. Macromol. Chem. Phys. 2011, 212, 2159-2165.

(37) Tait, J. G.; Merckx, T.; Li, W.; Wong, C.; Gehlhaar, R.; Cheyns, D.; Turbiez, M.; Heremans, P. Determination of Solvent Systems for Blade Coating Thin Film Photovoltaics. Adv. Funct. Mater. 2015, 24, 3393-3398.

(38) Burgués-Ceballos, I.; Machui, F.; Min, J.; Ameri, T.; Voigt, M. M.; Luponosov, Y. N.; Ponomarenko, S. a.; Lacharmoise, P. D.; Campoy-Quiles, M.; Brabec, C. J. Solubility Based Identification of Green Solvents for Small Molecule Organic Solar Cells. Adv. Funct. Mater. 2014, 24, 1449-1457.

(39) Walker, B.; Tamayo, A.; Duong, D. T.; Dang, X. D.; Kim, C.; Granstrom, J.; Nguyen, T. Q. A Systematic Approach to Solvent Selection Based on Cohesive Energy Densities in a Molecular Bulk Heterojunction System. Adv. Energy Mater. 2011, 1, 221-229.

(40) Baeg, K.-J.; Khim, D.; Jung, S.-W.; Koo, J. B.; You, I.-K.; Nah, Y.-C.; Kim, D.-Y.; Noh, Y.-Y. Polymer Dielectrics and Orthogonal Solvent Effects for High-Performance Inkjet-Printed Top-Gated P-Channel Polymer Field-Effect Transistors. ETRI J. 2011, 33, 887-896.

(41) Hildebrand, J. H.; Scott, R. L. The Solubility of Nonelectrolytes; Third.; Reinhold, New York, 1950.

(42) Hildebrand, J. H.; Scott, R. L. Regular Solutions; Prentice-Hall: Englewood Cliffs, NJ, 1962.

(43) Hansen, C. M. Hansen Solubility Parameters; 2nd ed.; CRC Press, 2007.

(44) Hansen, C. M. Aspects of Solubility, Surfaces and Diffusion in Polymers. Prog. Org. Coatings 2004, $51,55-66$.

(45) Hansen, C. M. 50 Years with Solubility Parameters - Past and Future. Prog. Org. Coatings 2004, 51, $77-84$.

(46) Boudinet, D.; Benwadih, M.; Qi, Y.; Altazin, S.; Verilhac, J. M.; Kroger, M.; Serbutoviez, C.; Gwoziecki, R.; Coppard, R.; Le Blevennec, G.; et al. Modification of Gold Source and Drain Electrodes by Self-Assembled Monolayer in Staggered N- and P-Channel Organic Thin Film Transistors. Org. Electron. 2010, 11, 227-237. 
(47) Noh, Y.-Y.; Sirringhaus, H. Ultra-Thin Polymer Gate Dielectrics for Top-Gate Polymer Field-Effect Transistors. Org. Electron. 2009, 10, 174-180.

(48) Kline, R. J.; DeLongchamp, D. M.; Fischer, D. A.; Lin, E. K.; Heeney, M.; McCulloch, I.; Toney, M. F. Significant Dependence of Morphology and Charge Carrier Mobility on Substrate Surface Chemistry in High Performance Polythiophene Semiconductor Films. Appl. Phys. Lett. 2007, 90, 2005-2008.

(49) Chabinyc, M. L.; Toney, M. F.; Kline, R. J.; McCulloch, I.; Heeney, M. X-Ray Scattering Study of Thin Films of poly(2,5-bis(3-Alkylthiophen-2-yl)thieno[3,2-B]thiophene). J. Am. Chem. Soc. 2007, 129, 3226-3237. 
A methodology for identifying orthogonal solvents for solution-processed organic field effect transistor is reported. Hildebrand and Hansen solubility theory is used to construct solubility boundary for commonly used organic semiconductors and polymer gate dielectrics. The effect of orthogonality of a polymer dielectric solution on the semiconductor morphology and electrical characteristics of OFETs is studied.

Keyword - (Organic Semiconductors, Orthogonal Solvents, Hansen Solubility, TIPS-pentacene, PBTTT)

Abhinav M. Gaikwad,* Yasser Khan, Aminy E. Ostfeld, Shishir Pandya, Sameer Abraham, and Ana Claudia Arias*

\section{Identifying Orthogonal Solvents for Solution Processed Organic Transistors}

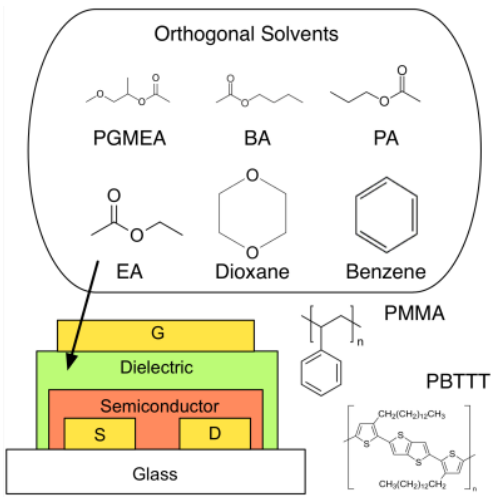

